\title{
CONDUTIVIDADE HIDRÁULICA DE UM LATOSSOLO ROXO, NÃO SATURADO, SOB DIFERENTES SISTEMAS DE USO E MANEJO ${ }^{1}$
}

\author{
UNSATURATED SOIL HYDRAULIC CONDUCTIVITY OF UN OXISOL, \\ UNDER DIFFERENT USE AND MANAGEMENT SYSTEMS
}

\author{
Vilson Antonio Klein ${ }^{2}$ Paulo Leonel Libardi ${ }^{3}$
}

RESUMO

O objetivo deste trabalho foi avaliar as alterações na condutividade hidráulica de um Latossolo Roxo ácrico (Typic Acrorthox) do município de Guaira, Região Norte do Estado de São Paulo, submetido ao plantio direto sem irrigação $e$ ao plantio direto irrigado comparando com o solo de mata, condição natural. Determinou-se a condutividade hidráulica do solo (K), no campo, em função do conteúdo de água $(\theta)$, pelo método do perfil instantâneo, avaliando-se a redistribuição da água no perfil do solo, por meio de tensiômetros instalados de 0,1 em $0,1 \mathrm{~m}$ até $1 \mathrm{~m}$ de profundidade. Para determinar a função $K(\theta)$ também em laboratório, amostras de solo com estrutura preservada, de 1,15m de comprimento e 0,2m de diâmetro, foram coletadas nos três manejos. Esses monólitos de solo foram instrumentados com tensiômetros e submetidos a variados fluxos estacionários descendentes de água, para determinação da função, pela equação de Darcy-Buckingham. Os resultados demonstraram que a estrutura do solo foi afetada pelos manejos até a profundidade de 0,4m, com maior intensidade no solo sob plantio direto irrigado, provocando redução da condutividade hidráulica do solo não saturado. $O$ movimento da água no solo não saturado também foi afetado pelo aumento da densidade, especificamente pela alteração na distribuição do diâmetro dos poros, demonstrando ainda que, com a metodologia adotada (campo e monólito) o solo, na condição natural, apresenta duas fases distintas com relação à condução de água, uma próxima à saturação e outra a partir da tensão de $40 \mathrm{kPa}$, pelo que as equações de $K(\theta)$ não podem ser extrapoladas para fora da faixa do conteúdo de água em que elas foram obtidas.

Palavras-chave: manejo do solo, dinâmica da água no solo, plantio direto.

\section{SUMMARY}

The objective of this paper was to assess the changes of the soil hydraulic conductivity of an oxisol (Typic Acorthox) of the county of Guaira (SP), Brazil (20⒉' $\left., 48^{\circ} 18^{\prime} \mathrm{W}, 518 \mathrm{~m}\right)$ by comparing the managements of no-tillage-non-irrigated and notillage-irrigated with the natural condition (forestry). Soil hydraulic conductivity $K$ as a function of soil water content $\theta$ was determined in the field by means of the instantaneous profile method, by assessing the water redistribution in the soil profile, by using tensiometers installed at $0.10 \mathrm{~m}$ soil depth intervals from $0.10 \mathrm{~m}$ to $1.00 \mathrm{~m}$. The $K(\theta)$ function was also determined in the laboratory, using undisturbed soil samples of $1.15 \mathrm{~m}$ length and $0.20 \mathrm{~m}$ diameter; these samples or soil monoliths from the three treatments, were instrumented with tensiometers and submitted to several steady descendent water fluxes in the laboratory in order to determine the $K(\theta)$ functions by means of the DarcyBuckingham equation. Results have shown that the soil structure was affected by managements till the soil depth of $0.40 \mathrm{~m}$, more intensively in the soil under no-tillage-irrigated, leading to a decrease in the unsaturated soil hydraulic conductivity. The unsaturated soil water movement was also affected by the increase of the soil bulk density, due to the change on the soil pore diameter distribution. With the adopted methodology (field and soil monolith), it could be perceived that, under the natural condition, the studied soil presented two distinct phases with relation to soil water conduction, one near the saturation and other from the soil water content at tensions equal and higher than $40 \mathrm{kPa}$; this fact led to the conclusion that the $K(\theta)$ equations cannot be extrapolated to soil water content ranges out of those they were measured.

Key words: soil managament, soil water dynamics, no tillage.

\footnotetext{
${ }^{1}$ Extraído da Tese do Doutorado, apresentada pelo primeiro autor à Escola Superior de Agronomia Luis de Querioz (ESALQ), Universidade de São Paulo (USP), Piracicaba, SP.

${ }^{2}$ Engenheiro, Agrônomo. Doutor, Professor Faculdade de Agronomia e Medicina Veterinária, Universidade de Passo Fundo. CP. 611, 99001-970, Passo Fundo, RS. E-mail: vaklein@upf.tche.br Autor para correspondência.

${ }^{3}$ Engenheiro Agrônomo. Professor Titular DCE, ESALQ, USP. Pesquisador do CNPq. E-mail: pllibard@ ciagri.usp.br.
} 


\section{INTRODUÇÃO}

O fluxo da água em solo não saturado, na região do sistema radicular dos cultivos é muito pouco estudado. No entanto, o seu conhecimento é de fundamental importância no entendimento dos processos de infiltração, redistribuição e suprimento de água às culturas.

As propriedades físicas do solo, como a textura e a estrutura determinam o fluxo de água no solo. Nesse sentido, ELLIES et al. (1997) destacam a importância da funcionalidade do sistema poroso do solo, englobando propriedades tais como: quantidade, tamanho, morfologia, continuidade e orientação dos poros. Todas estas propriedades do espaço poroso, que influenciam a condutividade, podem ser reunidas no termo único "geometria porosa dos solos" (LIBARDI 1999).

A condutividade hidráulica $K$ do solo não saturado, pode ser descrita como uma função $K(\theta)$, onde $\theta=$ umidade volumétrica do solo, que traduz o quanto ele conduz de água em dada umidade. A contribuição que este fluxo pode representar para a região das raízes das plantas é, ainda, mais importante e foi estudado por JONG VAN LIER \& LIBARDI (1997) que observaram que uma raiz individual de uma planta de arroz é capaz de retirar água do solo de uma distância de mais de $0,1 \mathrm{~m}$ no intervalo de alguns dias. Esses resultados concordam com os de HULUGALLE \& WILLATT (1983) que destacam que, em condições de baixa densidade de raízes, deve-se utilizar uma condutividade hidráulica crítica em vez de uma umidade crítica na avaliação do desenvolvimento das plantas.

Segundo SHARMA \& UEHARA (1968), a condutividade hidráulica, para Latossolos, decresce rapidamente, quando pequenas tensões são aplicadas. Próximo à saturação, a condutividade foi de aproximadamente $150 \mathrm{~mm} \cdot \mathrm{h}^{-1}$, com $10 \mathrm{kPa}$ de tensão foi reduzida para $0,1 \mathrm{~mm} \cdot \mathrm{h}^{-1}$. OTHMER $\boldsymbol{e t} \boldsymbol{a l}$. (1991) atribuem isto à agregação desse solo, que proporciona uma distribuição bimodal do diâmetro dos seus poros os quais são, por isso, classificados em poros inter-agregados e poros intra-agregados. Os primeiros são rapidamente esvaziados fazendo decrescer rapidamente a condutividade, e os segundos, mais lentamente, proporcionando uma condutividade menor.

O comportamento da condutividade hidráulica, em solos com estrutura microagregada próximo a saturação é diferente de outros solos argilosos (RADULOVICH $\boldsymbol{e} \boldsymbol{t} \boldsymbol{a l}$., 1992 e CHEN $\boldsymbol{e t}$ al., 1993). Nesses solos, na faixa de umidade de domínio dos macroporos (poros interagregados), a função $K(\theta)$ é governada pelo potencial gravitacional, enquanto na faixa de umidade abaixo da capacidade de campo (poros intra-agregados) o potencial mátrico passa a governar, valendo para esta condição a clássica teoria, baseada na equação de Richards.

Estudando as mudanças nas características físicas do solo sob diferentes manejos, BLEVINS et al. (1984) concluíram que a condutividade hidráulica do solo não saturado, em uma faixa de baixas tensões, foi maior para solos sob plantio direto do que em sistema de cultivo convencional. Em contraposição, ROTH et al. (1988) estudando os parâmetros físicos e hídricos do solo em diferentes manejos, constataram que em potenciais abaixo de $20 \mathrm{kPa}$, o plantio direto apresentou menores valores de condutividade hidráulica do que manejo convencional, enquanto, acima deste potencial mátrico, apresentou valores superiores, o que segundo os autores é muito importante no contexto da determinação de água disponível às plantas. Esta aparente contradição pode ser explicada em função de alguns fatores, entre os quais a variação de manejos adotados dentro do plantio direto, uns com maior atividade biológica no solo, outros utilizando culturas com sistema radicular mais agressivo e profundo, e a própria variação das características físicas do solo.

O objetivo deste trabalho foi determinar a função $K(\theta)$ de um Latossolo roxo, sob três condições de uso e manejo: plantio direto sem irrigação, plantio direto irrigado e mata natural.

\section{MATERIAL E MÉTODOS}

A área experimental localiza-se no Município de Guaira, situado na Região Norte do Estado de São Paulo, a qual é muito expressiva em irrigação por aspersão, tendo atualmente em torno de 200 pivôs centrais. O solo da área estudada é o Latossolo Roxo ácrico, A moderado, textura argilosa, profundo, bem drenado em função da sua microagregação e ocupando $85 \%$ da superfície do município (TOGNON, 1991; IPT, 1995).

Para avaliar o efeito dos manejos de solo sobre as propriedades físicas e hídricas do solo, três sistemas de uso e manejo foram selecionados, situados em áreas adjacentes. A primeira, com vegetação de mata secundária, não sofreu quaisquer tipos de cultivo e manejo. Suas propriedades físicos e hídricas foram consideradas como referência para comparação com os outros sistemas.

O solo sob condições de sequeiro situa-se em uma área desmatada em 1959, que desde então vem sendo cultivada com duas culturas anuais, uma no período chuvoso e outra na safrinha. Essa área até 
1991, foi cultivada no sistema convencional de preparo, com utilização de implementos de discos (arados e grades) e, a partir de então, passou a ser manejada de forma conservacionista, intercalando plantio direto e preparo reduzido.

A terceira área, com solo irrigado, foi desmatada em 1981, quando foi instalado o sistema de irrigação por pivô central, sendo cultivada desde então com culturas anuais em sistema de manejo conservacionista, com plantio direto intercalado com aração profunda, quando da instalação da cultura do tomate (aração profunda em 1990 e 1992).

Para determinar as alterações na estrutura do solo em profundidade, amostras com estrutura preservada foram coletadas, por meio de anéis volumétricos, os quais foram submetidos a diferentes tensões utilizando-se funis de placa porosa e câmaras de Richards. A porosidade foi classificada em função do diâmetro dos poros. Para esta classificação, chamouse de "macroporos", poros com diâmetro maior que $0,05 \mathrm{~mm}$ (que perdem a água em tensões menores que $6 \mathrm{kPa}$ ), "microporos" aqueles com diâmetro entre $0,05 \mathrm{e}$ $0,0002 \mathrm{~mm}$ (que são esvaziados a tensões entre 6 e $1500 \mathrm{kPa}$ ) e "criptoporos", poros com diâmetro menor que $0,0002 \mathrm{~mm}$ (que perdem a água apenas para tensões maiores que $1500 \mathrm{kPa}$ ).

Para determinação, a campo, da condutividade hidráulica do solo não saturado, nove baterias de 10 tensiômetros (três em cada tratamento) foram instaladas, nas profundidades de $0,1 \mathrm{em} 0,1 \mathrm{~m}$ até $1 \mathrm{~m}$, próximo ao local da abertura das trincheiras onde foram coletadas as amostras.

Utilizou-se o método do perfil instantâneo proposto por HILLEL et al. (1972). Para proceder à inundação das parcelas, utilizaram-se folhas de flandre e, unindo as extremidades por meio de parafusos e vedação de borracha, obteve-se um círculo com $5 \mathrm{~m}$ de diâmetro, correspondendo a $19,60 \mathrm{~m}^{2}$ de área inundada. Abriu-se um sulco em forma circular, com $0,2 \mathrm{~m}$ de profundidade, onde foi introduzida a folha de flandre. Após fechado o sulco, iniciou-se a inundação das parcelas, utilizando-se em torno de $10 \mathrm{~m}^{3}$ de água. Quando as leituras nos tensiômetros acusavam a saturação do solo até a profundidade de $1,0 \mathrm{~m}$, cessava-se a inundação e, para evitar evaporação de água, cobriu-se a parcela com duas lonas de plástico, e com folhas de isopor junto aos tensiômetros, para evitar a variação da temperatura que provocaria a condensação à noite.

No tempo zero de redistribuição, iniciaram-se as leituras dos tensiômetros e após, na primeira hora da manhã, antes de o sol aquecer o plástico e a água nos tensiômetros, efetuavam-se as leituras do potencial mátrico durante um período de aproximadamente 30 dias.
Com os valores do potencial mátrico e das curvas de retenção, para cada camada de solo, gráficos do armazenamento da água no solo em função do tempo para cada profundidade e manejo do solo, foram ajustados aos dados à uma equação do tipo potencial.

$$
\mathrm{h}_{\mathrm{z}}=\mathrm{at} \mathrm{t}^{\mathrm{b}}
$$

onde: $\mathrm{h}_{\mathrm{z}}=$ lâmina da água armazenada no solo $(\mathrm{mm})$ até a profundidade $\mathrm{z}(\mathrm{m}) ; \mathrm{t}=$ tempo(h); $\mathrm{a}\left(\mathrm{mm} \mathrm{h}^{-\mathrm{b}}\right) \mathrm{e}$ b são os parâmetros empíricos.

Derivando-se a equação 1 com relação ao tempo, obtém-se a equação 2 para cálculo do fluxo (q) de água em cada profundidade e tempo.

$$
\frac{d h z}{d t}=\mathrm{q}=\mathrm{ab} \mathrm{t} \mathrm{t}^{\mathrm{b}-1}
$$

Para o cálculo do gradiente de potencial total, obtido através dos tensiômetros, foram utilizados os valores de potencial total dos limites da camada de solo considerada (equação 3), razão pela qual não foi possível calcular a condutividade na primeira e última profundidades.

$$
\operatorname{grad} \phi t\left(Z_{i}\right)=\frac{\phi t\left(Z_{i-1}\right)-\phi t\left(Z_{i+1}\right)}{Z_{i-1}-Z_{i+1}}
$$

Calculou-se a condutividade hidráulica do solo não saturado, através da equação:

$$
K(\theta)=\underset{\operatorname{grad} \phi}{\mathrm{q}} \overline{ }
$$

Para a obtenção de uma relação entre $\mathrm{K}$ e $\theta$ procurou-se ajustar os valores de $K(\theta)\left(\mathrm{mm}^{-1} \mathrm{~h}^{-1}\right)$ em função de $\theta\left(\mathrm{m}^{3} \cdot \mathrm{m}^{-3}\right)$, para cada profundidade e manejo de solo (equação 5).

$$
\ln \mathrm{K}=\mathrm{a} \theta+\mathrm{b}
$$

onde: $\mathrm{K}=$ condutividade hidráulica $\left(\mathrm{mm} \cdot \mathrm{h}^{-1}\right) ; \theta=$ conteúdo de água no solo $\left(\mathrm{m}^{3} \cdot \mathrm{m}^{-3}\right)$; a e $\mathrm{b}=$ parâmetros empíricos.

Para determinar a condutividade hidráulica do solo não saturado em laboratório, amostras com estrutura preservada foram coletadas utilizando-se tubos de PVC (polivinil-cloreto) de $1,15 \mathrm{~m}$ comprimento e $0,2 \mathrm{~m}$ de diâmetro e com parede de $4,5 \mathrm{~mm}$. Utilizando-se uma estrutura de madeira, ancorada ao solo por meio de estacas de ferro e arames, que serviu de apoio a um macaco hidráulico com capacidade de $20 \mathrm{kN}$, introduziu-se o tubo no solo.Conforme o tubo era cravado no solo, 
cavava-se em torno do mesmo, para facilitar a penetração e a retirada da amostra. Essa coluna foi instrumentada com aplicador de água e tensiômetros. Para determinar a condutividade hidráulica, promoviam-se diferentes fluxos descendentes de água através da coluna, o que era obtido com um sistema de gotejamento na superfície do solo. Esperava-se ocorrer fluxo estacionário, que era medido com uma proveta, em um dispositivo de nível constante, conectado ao aplicador de água da parte inferior da coluna.

\section{RESULTADOS E DISCUSSÃO}

\section{Determinação a campo}

Para o fluxo de água no solo (q), nas diferentes profundidades e tempos, calculado pela equação 2, observa-se uma maior depleção de água na camada superficial para o solo da mata no tempo inicial (até 48 horas) em comparação aos outros dois manejos (Tabela 1). Em termos de manejo da irrigação, isto pode ser considerado um fator negativo, pois nessa condição natural, em função da pouca capacidade de retenção, irrigações mais freqüentes e com menor lâmina deveriam ser aplicadas, apresentando esse solo argiloso comportamento semelhante a um solo arenoso.

$\mathrm{Na}$ tabela 2, estão apresentados os parâmetros empíricos do ajuste de $\mathrm{K}$ em função de $\theta$ pela equação 5. Ajustou-se uma única equação aos dados experimentais para as três repetições por profundidade. Observa-se que o ajuste foi satisfatório $(\mathrm{p}<0,05)$, e $\mathrm{o}$ coeficiente de determinação foi superior a 0,79 , indicando não haver para esta propriedade do solo uma variabilidade espacial grande, ao contrário do que constataram REICHARDT et al. (1993) para uma Terra Roxa estruturada em Piracicaba (SP), o que talvez possa ser atribuído a maior variabilidade espacial daquele solo e ao equipamento usado para determinar o conteúdo de água no solo, que foi a sonda de nêutrons.

Analisando-se os parâmetros de ajuste do $\mathrm{K}$ em função de $\theta$ (Tabela 2 ) pode-se constatar uma menor condutividade para o solo irrigado, até a profundidade de $0,5 \mathrm{~m}$, coincidindo com a camada em que ocorreram as maiores alterações na porosidade total e na distribuição do tamanho dos

Tabela 1 - Fluxo da água no solo no sentido descendente $\left(\mathrm{mm} \mathrm{dia}^{-1}\right)$, durante a redistribuição da água no solo, para os três manejos em profundidade, Piracicaba, 1998

\begin{tabular}{|c|c|c|c|c|c|c|c|c|}
\hline \multirow[b]{2}{*}{ Período } & \multicolumn{8}{|c|}{ Profundidade (m) } \\
\hline & 0,2 & 0,3 & 0,4 & 0,5 & 0,6 & 0,7 & 0,8 & 0,9 \\
\hline $\mathrm{h}$ & \multicolumn{8}{|c|}{ Mata } \\
\hline 12 & 8,399 & 13,053 & 17,121 & 39,816 & 29,328 & 29,583 & 34,734 & 31,777 \\
\hline 24 & 3,546 & 4,856 & 7,014 & 13,824 & 11,909 & 14,260 & 12,537 & 13,423 \\
\hline 48 & 1,673 & 2,361 & 3,773 & 5,232 & 5,026 & 6,792 & 5,651 & 7,686 \\
\hline 96 & 0,842 & 1,195 & 2,023 & 2,422 & 3,004 & 3,723 & 3,236 & 4,131 \\
\hline 192 & 0,434 & 0,693 & 0,905 & 1,237 & 1,840 & 1,952 & 2,207 & 2,257 \\
\hline 240 & 0,338 & 0,628 & 0,751 & 0,905 & 1,431 & 1,561 & 2,095 & 1,848 \\
\hline 480 & 0,204 & 0,273 & 0,341 & 0,400 & 0,720 & 1,362 & 1,435 & 0,993 \\
\hline 720 & 0,133 & 0,124 & 0,084 & 0,323 & 0,652 & 0,599 & 0,939 & 0,651 \\
\hline \multicolumn{9}{|c|}{ Plantio direto sem irrigação } \\
\hline 12 & 5,344 & 4,050 & 8,824 & 8,450 & 11,863 & 12,687 & 20,546 & 26,773 \\
\hline 24 & 3,026 & 2,102 & 5,065 & 4,700 & 6,189 & 7,180 & 11,002 & 13,175 \\
\hline 48 & 2,130 & 1,080 & 2,538 & 2,678 & 3,545 & 4,493 & 5,340 & 6,770 \\
\hline 96 & 1,414 & 0,609 & 1,611 & 1,800 & 2,068 & 3,104 & 3,332 & 4,031 \\
\hline 192 & 0,753 & 0,353 & 0,784 & 0,914 & 1,325 & 1,867 & 1,898 & 1,979 \\
\hline 240 & 0,555 & 0,310 & 0,672 & 0,851 & 1,204 & 1,223 & 1,481 & 1,742 \\
\hline 480 & 0,236 & 0,156 & 0,431 & 0,388 & 0,696 & 0,980 & 0,977 & 1,292 \\
\hline 720 & 0,038 & 0,118 & 0,278 & 0,256 & 0,466 & 0,699 & 0,608 & 0,784 \\
\hline \multicolumn{9}{|c|}{ Plantio direto com irrigação } \\
\hline 12 & 4,686 & 9,279 & 15,524 & 19,166 & 27,761 & 22,305 & 20,986 & 33,614 \\
\hline 24 & 2,189 & 3,362 & 7,128 & 6,973 & 9,901 & 10,972 & 11,123 & 11,824 \\
\hline 48 & 1,199 & 1,576 & 3,366 & 5,668 & 5,168 & 5,158 & 6,179 & 7,610 \\
\hline 96 & 0,687 & 0,737 & 1,830 & 2,973 & 2,735 & 2,848 & 3,556 & 4,456 \\
\hline 192 & 0,410 & 0,381 & 1,012 & 1,220 & 1,359 & 1,556 & 2,247 & 2,548 \\
\hline 240 & 0,326 & 0,284 & 0,878 & 0,906 & 0,974 & 1,440 & 2,011 & 1,971 \\
\hline 480 & 0,168 & 0,131 & 0,407 & 0,419 & 0,550 & 0,899 & 1,111 & 1,331 \\
\hline 720 & 0,142 & 0,073 & 0,252 & 0,281 & 0,432 & 0,695 & 0,747 & 0,970 \\
\hline
\end{tabular}


Condutividade hidráulica de um latossolo roxo, não saturado...

Tabela 2 - Parâmetros empíricos para cálculo da condutividade hidráulica do solo não saturado em função dos manejos, ajustados a partir de dados obtidos no campo ${ }^{1}$. Piracicaba, 1998.

\begin{tabular}{|c|c|c|c|c|c|c|c|c|c|}
\hline \multirow{2}{*}{ Prof. (m) } & \multicolumn{3}{|c|}{ Mata } & \multicolumn{3}{|c|}{ Sequeiro } & \multicolumn{3}{|c|}{ Irrigado } \\
\hline & $\mathrm{a}$ & $\mathrm{b}$ & $r^{2}$ & $\mathrm{a}$ & $\mathrm{b}$ & $r^{2}$ & $\mathrm{a}$ & $\mathrm{b}$ & $\mathrm{r}^{2}$ \\
\hline 0,2 & 53,47 & $-22,22$ & 0,85 & 30,95 & $-13,78$ & 0,79 & 59,56 & $-27,10$ & 0,94 \\
\hline 0,3 & 54,61 & $-21,61$ & 0,97 & 48,84 & $-20,02$ & 0,75 & 57,76 & $-27,47$ & 0,95 \\
\hline 0,4 & 54,30 & $-21,33$ & 0,91 & 47,26 & $-18,60$ & 0,94 & 46,99 & $-22,05$ & 0,93 \\
\hline 0,5 & 50,86 & $-20,98$ & 0,92 & 57,32 & $-22,42$ & 0,93 & 52,08 & $-22,56$ & 0,97 \\
\hline 0,6 & 35,32 & $-14,91$ & 0,88 & 37,92 & $-15,71$ & 0,91 & 59,33 & $-24,96$ & 0,93 \\
\hline 0,7 & 49,08 & $-19,52$ & 0,95 & 49,46 & $-19,16$ & 0,91 & 41,17 & $-16,96$ & 0,97 \\
\hline 0,8 & 33,38 & $-14,47$ & 0,92 & 40,87 & $-17,08$ & 0,96 & 43,16 & $-18,48$ & 0,89 \\
\hline 0,9 & 35,27 & $-15,65$ & 0,98 & 38,35 & $-15,23$ & 0,93 & 35,58 & $-15,00$ & 0,94 \\
\hline
\end{tabular}

${ }^{1}$ Parâmetros empíricos a e b da equação $\ln \mathrm{K}=\mathrm{a} \theta+\mathrm{b}$ e $\mathrm{r}^{2}=$ coeficiente de determinação.

poros em função da compactação do solo (Figura 1), concordando com ROTH et al. (1988) que também encontraram influência negativa sobre o $K(\theta)$ em função de manejos e cultivos sucessivos.

É importante destacar que a faixa de umidade $\left(0,45\right.$ a $\left.0,30 \mathrm{~m}^{3} \cdot \mathrm{m}^{-3}\right)$ na qual se determinou a condutividade hidráulica do solo não saturado no campo é relativamente estreita, devido à rápida drenagem da água no início da determinação e da elevada capacidade de retenção de água que este solo possui. No caso da mata, a maior parte da água drenou rapidamente, dificultando medidas iniciais em função do tempo de resposta dos tensiômetros, uma vez que a condutância hidráulica média das cápsulas porosas era de $1.10^{-5} \mathrm{~cm}^{2} . \mathrm{s}^{-1}$ demorando para atingir o equilíbrio. No sistema irrigado, em função da redução na porosidade do solo acarretada pelo aumento da densidade (Figura 1), a umidade estabilizou em valores bem mais altos drenando menor quantidade de água, permitindo efetuar as determinações em umidades próximas à saturação.

Para o tempo de 12 horas e profundidade de $0,2 \mathrm{~m}$ em solo de mata, a $\mathrm{K}(0,391)$ era de $6,45 \mathrm{~mm} \cdot \mathrm{dia}^{-1}$, enquanto no solo de cultivo irrigado, a $\mathrm{K}(0,425)$ era de $3,97 \mathrm{~mm} \cdot \mathrm{dia}^{-1}$, obtidas a partir da equação 5. Isto evidencia que, no sistema de cultivo irrigado, mesmo mantendo a umidade do solo mais elevada, a condutividade hidráulica do solo não saturado é menor. A constatação de que a condutividade hidráulica do solo não saturado é afetada pelo aumento da densidade em função da diminuição da porosidade e alteração na distribuição dos poros é muito importante e deve ser considerada em termos de manejo da água no solo, conforme também enfatizam HORTON et al. (1994).

\section{Determinação em laboratório}

A determinação da $K(\theta)$ em amostras com estrutura preservada coletadas no campo se mostrou uma metodologia simples, rápida de ser executada, permitindo avaliações em uma faixa de umidade diferente daquela obtida a campo $\left(0,60-0,45 \mathrm{~m}^{3} \cdot \mathrm{m}^{-3}\right)$, mais próxima à saturação do solo, condição importante para a boa compreensão do processo de infiltração da água no solo.

$\mathrm{Na}$ tabela 3, estão apresentados os parâmetros empíricos do ajuste de $\ln \mathrm{K}$ versus $\theta$, permitindo obter a função $K(\theta)$. Destaca-se que só foi possível determinar a $\mathrm{K}(\theta)$ em cinco profundidades $(0,2 ; 0,3 ; 0,4 ; 0,5 ; 0,6)$, pois a primeira $0,1 \mathrm{~m}$ foi utilizada para o cálculo do grad $\phi \mathrm{t}$ na profundidade $0,2 \mathrm{~m}(0,1-0,3 \mathrm{~m})$, enquanto os tensiômetros instalados nas profundidades 0,8 e $0,9 \mathrm{~m}$ não saíram da faixa quase saturada, devido ao nível constante de água estabelecido na parte inferior da coluna de solo.

Observou-se também, com essa metodologia, diferenças no comportamento da condutividade para os diferentes manejos e profundidades, com a mesma tendência observada na determinação a campo, tendo o sistema irrigado menores valores até a profundidade $0,5 \mathrm{~m}$.

\section{Análise conjunta de $K(\theta)$ realizada no campo e em laboratório}

Observando-se os parâmetros de ajuste a e b da equação 5 (Tabela 2 e 3) notam-se as diferenças entre os valores obtidos no campo e em laboratório (Figura 2).

Observou-se, neste trabalho, pela diferença nos valores do coeficiente angular das equações de ajuste (Tabela 2 e 3 ) a diferença no comportamento da condutividade hidráulica próxima à saturação, em relação à outra fase na qual o potencial mátrico governa o movimento. Estes dados corroboram com as afirmações de RADULOVICH et al., (1992) e CHEN et al., (1993) que descrevem este fenômeno. Observa-se que essa diferença no 


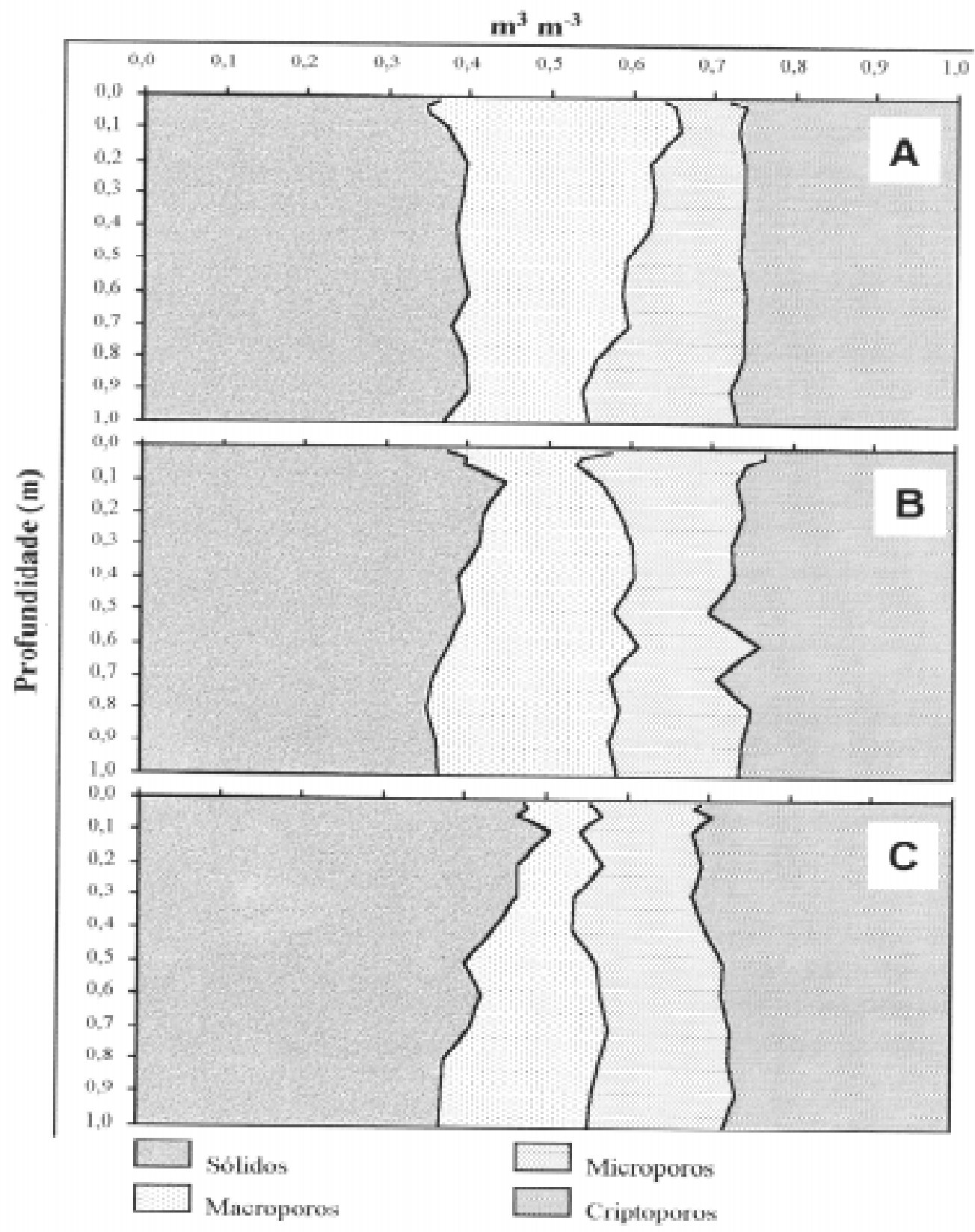

Figura 1 - Alterações na estrutura do solo, porosidade total e distribuição do diâmetro dos poros, em profundidade, para diferentes sistemas de uso e manejo ( $\mathrm{A}=$ mata; $\mathrm{B}=$ plantio direto sem irrigação e $\mathrm{C}=$ plantio direto irrigado).

comportamento foi diminuindo, à medida que, em função do aumento da densidade do solo (Figura 1), houve redução da macroporosidade. Na figura 2, observa-se como este efeito é acentuado no solo da mata, onde existe uma uniformidade de macroporos em grande volume em todo o perfil (Figura 1), enquanto no irrigado na camada até $0,4 \mathrm{~m}$ de profundidade, praticamente não se observa esse efeito.

A descontinuidade da função $K(\theta)$ não pode ser atribuída à utilização de diferentes metodologias, pois em várias profundidades observa-se que os valores de $\mathrm{K}$ obtidos em laboratório acompanham a tendência daqueles obtidos no campo. 


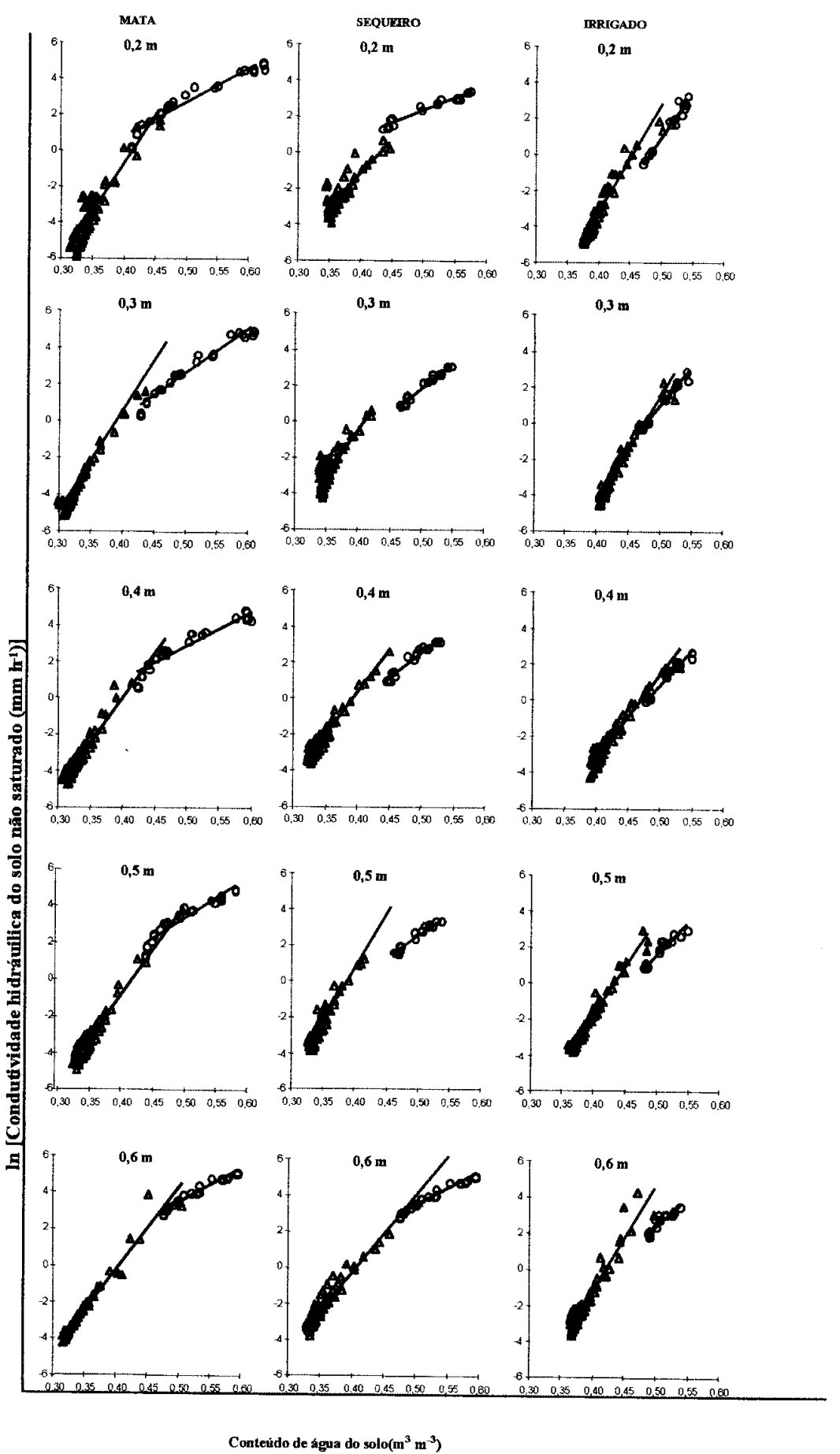

Figura 2 - Relação da condutividade hidráulica versus conteúdo da água do solo determinada no campo ( $\Delta$ ) e em laboratório (o), em profundidade, em um Latossolo roxo sob diferentes sistemas de uso e manejo. 
Tabela 3 - Parâmetros empíricos para cálculo da condutividade hidráulica do solo não saturado em função dos manejos, ajustados a partir de dados obtidos em laboratório ${ }^{1}$. Piracicaba, 1998.

\begin{tabular}{lccccccccc}
\hline & \multicolumn{3}{c}{ Mata } & \multicolumn{3}{c}{ Sequeiro } & \multicolumn{3}{c}{ Irrigado } \\
\cline { 2 - 9 } Prof. $(\mathrm{m})$ & $\mathrm{a}$ & $\mathrm{b}$ & $\mathrm{r}^{2}$ & $\mathrm{a}$ & $\mathrm{b}$ & $\mathrm{r}^{2}$ & $\mathrm{a}$ & $\mathrm{b}$ & $\mathrm{r}^{2}$ \\
\hline 0,2 & 19,287 & $-6,975$ & 0,930 & 14,112 & $-4,712$ & 0,955 & 48,961 & $-23,53$ & 0,965 \\
0,3 & 24,604 & $-9,819$ & 0,961 & 28,326 & $-12,34$ & 0,968 & 43,059 & $-20,56$ & 0,973 \\
0,4 & 19,125 & $-6,710$ & 0,908 & 27,577 & $-11,26$ & 0,945 & 39,146 & $-18,71$ & 0,953 \\
0,5 & 21,998 & $-7,687$ & 0,896 & 25,348 & $-10,16$ & 0,950 & 34,811 & $-15,81$ & 0,907 \\
0,6 & 18,538 & $-5,843$ & 0,961 & 30,525 & $-12,71$ & 0,924 & 31,53 & $-13,38$ & 0,864 \\
\hline
\end{tabular}

${ }^{1}$ Parâmetros empíricos a e b da equação $\ln \mathrm{K}=\mathrm{a} \theta+\mathrm{b}$ e $\mathrm{r}^{2}=$ coeficiente de determinaç̃ão.

Analisando o valor do conteúdo de água em que ocorre a mudança na inclinação das retas, em torno de $0,45 \mathrm{~m}^{3} \cdot \mathrm{m}^{-3}$ para todos os manejos, relacionando com a curva de retenção da água no solo, para obter potencial mátrico equivalente, calculou-se o tamanho aproximado do poro desta descontinuidade. O diâmetro situou-se em torno de 0,1mm concordando com LUXMOORE (1981) que sugere ser este o menor diâmetro dos poros para serem classificados como macroporos, responsáveis portanto pelo fluxo rápido da água no solo.

Isto sugere que a metodologia de determinação da condutividade hidráulica do solo não saturado a campo e em laboratório poderá ser utilizada para definir o limite do diâmetro dos poros no qual a ação da capilaridade (potencial mátrico) começa a interferir no movimento da água no solo.

\section{CONCLUSÕES}

Alterações na estrutura do solo, com aumento da densidade, redução da porosidade total e alteração na distribuição do diâmetro dos, diminuem a condutividade hidráulica de um Latossolo roxo não saturado.

O procedimento experimental tradicional do método do perfil instantâneo a campo não permite avaliar corretamente a função $K(\theta)$ para conteúdos de água do solo próximos à saturação.

A determinação da função $K(\theta)$ na faixa de conteúdo de água próximo à saturação no laboratório, usando-se amostras grandes de solo com estrutura preservada, é uma técnica que pode ser utilizada com sucesso para complementar os dados do método do perfil instantâneo a campo.

\section{REFERÊNCIAS BIBLIOGRÁFICAS}

BLEVINS, R.L., SMITH, M.S., THOMAS, G.W. Changes in soil properties under no-tillage. In: PHILLIPS, R.E., PHILLIPS, S.H. (Ed.) No-tillage agriculture: principles and practices. New York: VNR, 1984. Cap.9. p.190-230.
CHEN, C., THOMAS, D.M., GREEN, R.E., et al. Two-Domain estimation of hydraulic properties in macropore soils. Soil Science Society of America Journal, v.57, p.680-686, 1993.

ELLIES, A., GREZ, R., RAMIREZ, C. La conductividad hidráulica en fase saturada como herramienta par el diagnostico de la estructura del suelo. Agro Sur, v.5, p.51-56, 1997 .

HILLEL, D., KRENTOS, V.D., STYLIANOU, Y. Procedure and test of an internal drainage method for measuring soil hydraulic characteristics in situ. Soil Science, v.114, p.395400, 1972.

HORTON, R., ANKENY, M.D., ALLMARAS, R.R. Effects of compaction on soil hydraulic properties. In: SOANE, B.D., VAN OUWERKERK, C. (Ed.). Soil compaction in crop production. Amsterdam : Elsevier, 1994. Cap.7, p.141-165.

HULUGALLE, N.R., WILLATT, S.T. The role of soil resistance in determining water uptake by plant toot systems. Australian Journal of Soil Research, v.21, p.571-574, 1983.

INSTITUTO DE PESQUISAS TECNOLÓGICAS DO ESTADO DE SÃO PAULO. Áreas de conflito de uso das terras do município de Guaira - SP. São Paulo, 1995. 60p. (IPT. Relatório Técnico, 33 042).

JONG VAN LIER, Q. de, LIBARDI, P.L. Extraction of soil water by plants: development and validation of a model. Revista Brasileira de Ciência do Solo, v.212, p.535-542, 1997.

LIBARDI, P.L. Dinâmica da água no solo. 2.ed. Piracicaba : O autor, 1999. 497p.

LUXMOORE, R.J. Micro-,meso-, and microporosity of soil. Soil Science Society of America Journal, v.45, p.671-672, 1981.

OTHMER, H., DIEKKRUGER, B., KUTILEK, M. Bimodal porosity and unsaturated hydraulic conductivity. Soil Science, v.52, p.139-150, 1991.

RADULOVICH, R., SOLLINS, P., BAVEYE, P., et al. Bypass water flow through unsaturated microaggregated tropical soils. Soil Science Society of America Journal, v.56, p.721726, 1992.

REICHARDT, K., BACCHI, O.O.S., VILLAGRA, M.M., et al. Hydraulic variability in space and time in a dark red latosol on the tropics. Geoderma, v.60, p.159-168, 1993. 
ROTH, C.H., MEYER, B., FREDE, H.G., et al. Effect of mulch rates and tillage systems on infiltrability and other soil physical properties of an oxisol in Paraná, Brazil. Soil \& Tillage Research, v.11, p.81-91, 1988.

SHARMA, M.L., UEHARA, G. Influence of soil structure on water relations in low Humic Latosols: II. Water movement.
Soil Science Society of America Proceedings, v.32, p.770774, 1968.

TOGNON, A.A. Propriedades físico-hídricas do Latossolo Roxo da região de Guaira-SP sob diferentes sistemas de cultivo. Piracicaba, 1991. 85p. Dissertação (Mestrado em Solos e Nutrição de Plantas) - Escola Superior de Agricultura "Luiz de Queiroz", Universidade de São Paulo,1991. 